\title{
ETHzürich
}

ETH Library

\section{Whole joint structure in a rat osteoarthritis model for sample- specific mechanical evaluation}

\section{Conference Poster}

Author(s):

Steiner, Thomas H.; Choo, R.J.; Quintin, A.; Müller, Ralph (D); Zulliger, M.A.; Nesic, D.; Stok, K.S.

Publication date:

2012

Permanent link:

https://doi.org/10.3929/ethz-a-007350312

Rights / license:

In Copyright - Non-Commercial Use Permitted 
WHOLE JOINT STRUCTURE IN A RAT OSTEOARTHRITIS MODEL FOR SAMPLE-SPECIFIC MECHANICAL EVALUATION

\author{
Steiner, T.H. ${ }^{1}$, Choo, R.J. ${ }^{1}$, Quintin, A. ${ }^{2}$, Müller, R. ${ }^{1}$, Zulliger, M. A. ${ }^{3}$, Nesic, D. ${ }^{2}$, Stok, K.S. ${ }^{1}$
}

1. Institute for Biomechanics, ETH Zurich, Switzerland

2. Department of Clinical Research, University of Bern, Switzerland

3. SCANCO Medical AG, Brüttisellen, Switzerland

Introduction

Methods

Results and Discussion

Conclusions and Outlook

References

Acknowledge
Micro-computed tomography (microCT) allows 3D assessment of calcified tissue structures in the joint, and recent advances use contrast agents to quantify glycosaminoglycan content in cartilage [Palmer 2006], alongside measurement of volume changes [Siebelt 2011]. However, challenges in clearly delineating the cartilage from the surrounding joint space still remain. Solving this would allow more accurate definitions of structural requirements for finite element (FE) studies of mechanical loading, which is an important determinant in the progression of osteoarthritis $(\mathrm{OA})$.

The goal of this work is to provide accurate structural definitions for future FE studies, by clearly delineating the joint tissues using a single imaging modality; i.e. microCT.

Medial meniscectomy/ACL desmotomy of four 3 month-old male Wistar rats was performed to destabilise the right tibiofemoral joint. Seven non-operated rat joints served as healthy controls. Animals were sacrificed 6 weeks post-op, the knees dissected, and scanned using microCT (SCANCO Medical, Switzerland; $10 \mu \mathrm{m}$ voxel size). After a pre-scan, the joint space was filled with a contrast agent and immersed overnight in $60 \%$ Hexabrix $^{\mathrm{TM}}$ (Mallinckrodt, USA), followed by a second scan with the same settings. MicroCT scans were processed and the cartilage was manually segmented. Morphometric analysis was performed to obtain the average 3D cartilage thickness $(\mathrm{Cg} . \mathrm{Th})$ for the medial and lateral tibial plateaux. The joints were then sliced and stained with Safranin O. Registration of the bones from histology and microCT allowed identification of the microCT slice corresponding to the histology section (figure 1 a-c). Cartilage was then segmented from both images, and 2D Cg.Th was calculated and correlated (figure $1 \mathrm{~d}-\mathrm{e}$ ).

The results show a strong correlation between 2D Cg.Th measured from histology and the registered microCT slices (figure 2a). Correlations were similar for OA-induced and control rat knees, therefore the data was pooled to give a correlation, $R^{2}=0.80, p<0.001$

The protocol also provided a qualitative 3D assessment of cartilage, as shown in figure $2(b-c)$. This allows investigation of patterns of destruction in $\mathrm{OA}$, where the medial tibial plateaux showed cartilage loss and fibrillation compared to both the lateral plateaux and the healthy controls.

Finally, with segmentation of tibio-femoral components, it was also possible to generate an individualised joint model for FE analysis (figure 3); where the interface between tissues can be accurately defined, and sample-specific structural changes can be included.

In conclusion, the results clearly show a good correlation with quantitative cartilage measures taken from microCT compared to histomorphometry; which highlights the capacity of microCT to accurately measure cartilage structures. Furthermore, the high-resolution, 3D data obtained with microCT allows whole-joint analysis and imaging of diseaseinduced changes, and permits investigation of local bone adaptation alongside cartilage degeneration. The validated individual models produced with this technique can be used in future to study load bearing of articulating surfaces, and the role of microstructural changes in the progression of $O A$. Structural delineation also allows investigation of patterns of destruction in the onset of $\mathrm{OA}$ that can be used for individualised FE analysis of a particular joint of interest.

[1] Palmer AW, et al. PNAS, 103 (51): 19255-60, 2006. [2] Siebelt M, et al. J Orthop Res 29 (11): 1788-94, 2011.

Thanks to Dr. Markus Wilke for performing the animal surgeries, and Andreas Trüssel for his registration algorithm.

Funding: Swiss Commission for Technology and Innovation, 9853.1.
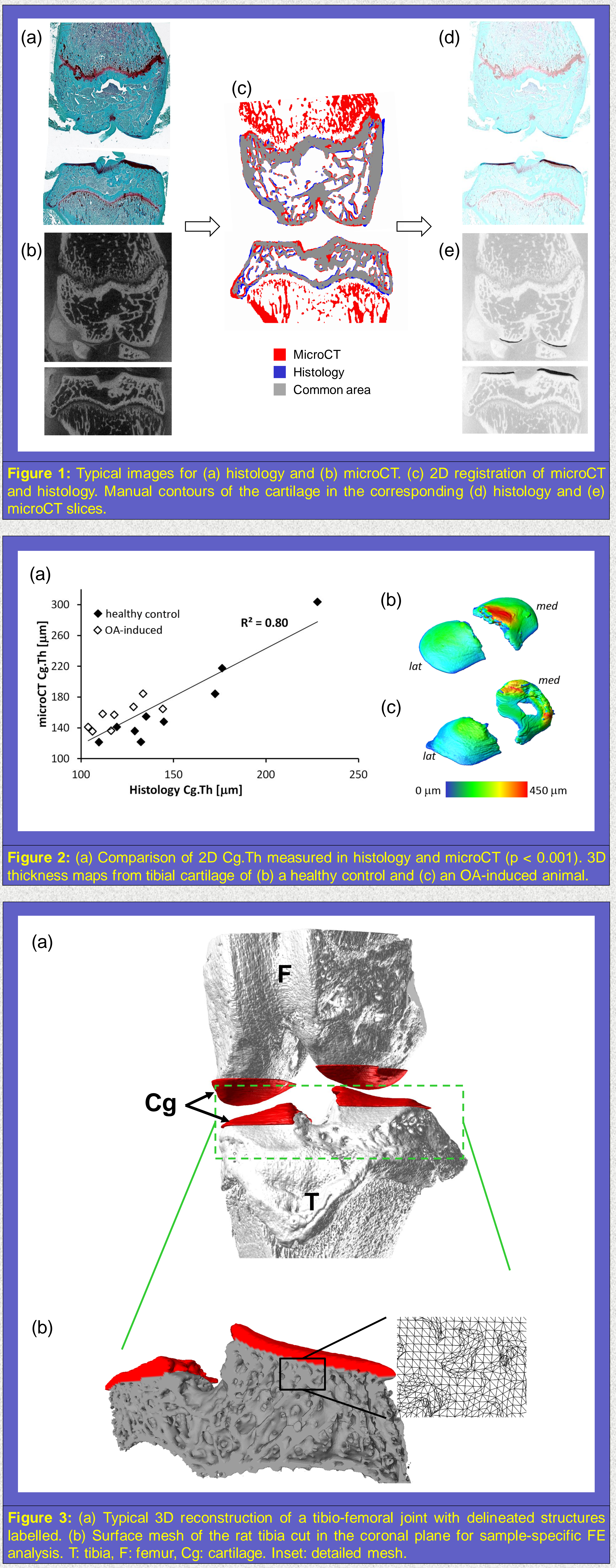